\title{
The unique role of nephrologists in the pursuit of global health goals
}

\author{
Kiyoshi Kurokawa
}

Nephrologists are at the front line of global efforts to stem the spread of chronic kidney disease (CKD). CKD affects millions of patients worldwide, many of whom require the costly treatments of chronic dialysis and transplantation. In addition, CKD is a major risk factor for cardiovascular disease and death. As a result of the increasing prevalence of conditions that predispose individuals to CKD, such as hypertension, diabetes and other lifestyle-related diseases, the prevention and early detection of CKD has become a health-care priority in both affluent and developing countries. Effective prophylactic and therapeutic regimens are well established, but the capacity of nephrologists to implement such regimens and the adherence of patients to these regimens depend, in part, upon the health policy of each country. The World Kidney Day initiative, launched in 2006, aims to put CKD on the agenda of organizations that determine health policy.

In addition to the treatment and prevention of CKD, there are many other pressing global issues to which the nephrology community can provide an important contribution. This year is the mid-term of the UN Millennium Development Goals (MDGs) initiative (http://www.un.org/ millenniumgoals), which was launched in 2000 with the aim of achieving eight global development goals by 2015 . Of these goals, four are directly related to health or medical issues: eradicating hunger, improving maternal health, lowering child mortality, and decreasing the prevalence of major diseases such as HIV/AIDS and malaria. Indeed, health-care issues have been a mainstay of the global agenda in the past few years, as demonstrated by the introduction of other initiatives, such as the WHO's Commission on Social Determinants of Health (http://www.who.int/social_determinants/en) and the Bill \& Melinda Gates Foundation (http:// www.gatesfoundation.org/default.htm).

I believe that there are several reasons why nephrologists are ideally placed to contribute to the achievement of the health-care MDGs. ....there

are several

reasons why

nephrologists

are ideally

placed to

contribute

to the

achievement

of the health-

care MDGs

[Millennium

Development

Goals]

K Kurokawa is Science Advisor to the Cabinet of the Japanese

Government, Professor

of the National

Graduate Institute for

Policy Studies, and

Chair of the Health

Policy Institute of

Japan, Tokyo, Japan.

Competing interests

The author declared no

competing interests.

www.nature.com/clinicalpractice doi:10.1038/ncpneph0893
Nephrology is a uniquely cross-sectional discipline of medicine. Nephrologists see patients with conditions that encompass a variety of subspecialties, including infectious diseases, gastroenterology, and cardiology. Some of the conditions associated with rapid decline of kidney function, such as fluid, electrolyte and acid-base disorders, are not well understood by other specialists. Care of patients with endstage renal disease involves dietary modification and monitoring of mineral metabolism parameters, and it forms a major part of the service provided by nephrologists in developed countries. Thus, many nephrologists are well versed in issues of nutrition and mineral metabolism.

In order to ensure that the unique expertise of nephrologists is available to patients in all parts of the world, it would be useful for nephrologists from developed countries, particularly those who are in training, to spend some time (perhaps 3-6 months or more) practicing their profession in impoverished and developing regions. In this way, the nephrologists of the future would experience first-hand the challenges that face global health. Through such experiences, these individuals would develop leadership skills and closer relations with local people, thus helping to advance the cause of the MDGs.

More than a decade ago, the International Society of Nephrology launched the COMGAN (Commission for the Global Advancement of Nephrology) initiative, which coordinates exchange programs between developed and developing countries at various levels. These programs are aimed at promoting partnerships and fostering the development of nephrology expertise. Instigation of similar programs outside the nephrology arena could contribute to the achievement of the health-related MDGs. The nephrology community should make the promotion of health in poverty-stricken regions of the world part of its agenda-perhaps as part of COMGAN. Reducing preventable morbidity and mortality in such regions is a major global concern. 